\title{
Der Homo oeconomicus: besser als sein Ruf!
}

0 Von Remco Feskens und Seyno van Es ft wird angenommen, dass Kooperation mit anderen und die Verfolgung der eigenen Interessen einander ausschließen. Dieser Gedanke erscheint auch in dem Artikel von Simon Gächter „Kein unlösbares Dilemma“ in der vorigen Ausgabe von Ökologisches Wirtschaften (1). Danach sind viele Menschen von Natur aus kooperativ, aber es gibt auch Trittbrettfahrer, also Akteure die nur von kooperativen Akteuren profitieren wollen. Durch diese Trittbrettfahrer würde keine Zusammenarbeit entstehen, obwohl dies gesamtgesellschaftlich gesehen das beste Ergebnis wäre. In einem anderen Artikel derselben Ausgabe zeigt Martin Beckenkamp, wie sich Kooperation sozialpsychologisch erklären lässt (2).

Unsere Thesen sind hingegen, dass

1. durch die Einführung von Sanktionen wie bei Gächter die Struktur der Präferenzen so verändert wird, dass das Modell kein soziales Dilemma mehr widerspiegelt und dass

2. Zusammenarbeit und Eigeninteresse durchaus Hand in Hand gehen können und sich damit Kooperation nicht nur sozialpsychologisch, sondern auch ökonomisch rational erklären lässt.

\section{- Trittbrettfahren}

Trittbrettfahren kann in einer Situation vorkommen, in der es unmöglich ist, Akteure von der Nutzung eines von anderen produzierten Guts auszuschließen. Diese Situation entsteht oft bei Gemeingütern, wie zum Beispiel saubere Luft.

Das ist auch die Ausgangsposition des ersten von Gächter vorgestellten Experiments. Wie bei einem Gefangenendilemma zu erwarten, ist das Ergebnis dann auch, dass viel weniger investiert wurde als sozial wünschenswert war. Dann wurde das Spiel geändert. Nach jeder Spielrunde gab es jetzt eine Sanktionsmöglichkeit. Akteure die viel investiert hatten, konnten Trittbrettfahrer jetzt bestrafen. Das Ergebnis war, dass die Investitionen viel höher waren als im ersten Experiment. Hieraus schließt Gächter, dass Personen im Prinzip kooperativ sind, falls Trittbrettfahrer ausgeschaltet werden. Dieses Experiment beweist jedoch nicht, dass hier die Rede von uneigennützigem Handeln sein kann. Die verfïgbaren Strategien der Akteure sind durch die Sanktionsmöglichkeiten verändert, dass aus rationalen egoistischen Motiven kooperiert wird: es lohnt sich nicht mehr, nicht zusammen zu arbeiten. Damit ist jedoch die Struktur des Experiments entscheidend verändert worden und spiegelt nicht mehr die Struktur eines sozialen Dilemmas wider. Oft werden, beispielsweise von Ostrom, auch Normen als Erklärung für das Erreichen von Zusammenarbeit in einem sozialen Dilemma angeführt (3). Aber auch in diesem Fall besteht eigentlich kein Unterschied mehr zwischen individueller und kollektiver Rationalität.

\section{- Rationale egoistische Zusammen- arbeit in sozialen Dilemmata}

Eine andere Sicht auf diese strategischen Handlungen erhält man, wenn man diese als wiederholte Interaktionen betrachtet. Bei dieser Variante wird versucht Voraussetzungen zu identifizieren, unter denen rationale egoistische Personen selbständig zur Kooperation gelangen. Ein Gedanke, den Menschen auch intuitiv erfassen: Personen verhalten sich oft kooperativer wenn sie wissen, dass sie ihre Gegenspieler in der Zukunft wiedertreffen werden. Beckenkamp präsentiert eine sozialpsychologische Erklärung für bedingte Kooperation in einem solchen Fall. Er zeigt, dass verschiedene psychologische Betrachtungen relevant sein können in der Analyse von Gemeingutkonflikten. Es gibt hierfür aber auch eine ökonomisch rationale Erklärung.

Wenn eine Interaktion nur einmalig statt findet, ist deutlich, dass Nicht-Kooperation die einzige rationale Strategie ist, was aber zu einem sozial unerwünschten Ergebnis führt. In einem wiederholten Spiel jedoch werden Akteure versuchen, ein kooperatives Klima zu schaffen. Jetzt zählen nicht nur die Ergebnisse des ersten Spiels, sondern werden auch zukünftige Ergebnisse miteinbezogen. Die Zukunft wirft ihren Schatten auf die heutigen Entscheidungen (3). Daher werden die Ergebnisse zukünftiger Spiele abdiskontiert in die Wahl einer Strategie einfließen. Neben einer ausreichend günstigen Zukunftserwartung ist die Form der Strategie wichtig. Wenn man entschieden hat zu kooperie- ren, ist es wichtig eine Strategie zu haben die auf Trittbrettfahrer reagiert. Eine solche Strategie, ist „Tit for Tat" (TFT). Sie besteht darin zu kooperieren, aber damit aufzuhören, wenn der Gegner nicht kooperiert. Wenn aber der Gegner (wieder) kooperiert, wird mit TFT auch wieder kooperiert. Diese bedingte TFT-Strategie ist, wie Michael Taylor auch formal gezeigt hat, in Kombination mit einer bestimmten Zukunftserwartung auch in einem Dilemma mit n Personen eine rationale Wahl (4).

Diese Strategie ist erfolgreich, weil sie eine transparente, nicht nachtragende, reaktive, aber vor allem eine kooperative Strategie ist. So kann es durchaus möglich sein, dass rationale Egoisten in demselben Dilemma-Spiel, welches allerdings mehrfach gespielt wird, zusammenarbeiten und so ein gesellschaftlich besseres Ergebnis erreichen.

Die Problematik der Gemeingüter wird oft als Legitimation für staatliche Intervention verwendet. Wenn aber Personen selbständig in der Lage sind, eine Lösung zu erreichen, ist diese zu bevorzugen, weil diese effizienter und mit höherer Akzeptanz der Bevölkerung zu Stande kommt, weil sie eben von den Betroffenen selbst initiiert worden ist. Auch wenn Individuen als Homo oeconomicus agieren, sind sie in der Lage, bei wiederholten Interaktionen durch entsprechende Strategien auch ohne staatlichen Eingriff einen Ausweg aus sozialen Dilemmata zu finden. Voraussetzungen sind, dass sie über gute Informationen über die Zukunft verfïgen, da die Zukunftserwartungen eine ausreichende starke Rolle spielen müssen um ein kooperatives Klima zu entstehen lassen. Zudem dürfen die negativen Folgen der NichtKooperation nicht zu weit in der Zukunft liegen, weil sie sonst wegdiskontiert werden.

\section{Anmerkungen}

(1) Gächter, S.: Kein unlösbares Dilemma, Ökologisches Wirtschaften, 2002, 6, S. 12-14.

(2) Beckenkamp, M.: Zug um Zug die Effizienz steigern, Ökologisches Wirtschaften, 2002, 6, S. 14-16.

(3) Ostrom, E.: Governing the Commons. The evolution of institutions for collective action, 1990, S. 193.

(4) Axelrod, R.: The Evolution of Cooperation, 1984.

(5) Taylor, M.: The Possibility of Cooperation, 1987.

\section{Die Autoren}

Remco Feskens ist Politikwissenschaffler und studiert zurzeit Statistik in Berlin, Seyno van Es ist Volkswirt. Kontakt: Remco Feskens, Dortmunder Straße 13, 10555 Berlin. Tel. 030-39746564,

E-Mail: remcofeskens@hotmail.com 
(c) 20I0 Authors; licensee IÖW and oekom verlag. This is an article distributed under the terms of the Creative Commons Attribution Non-Commercial No Derivates License (http://creativecommons.org/licenses/by-nc-nd/3.o/), which permits unrestricted use, distribution, and reproduction in any medium, provided the original work is properly cited. 